\title{
Des chimères du monde juridico- pénitentiaire au «Pégase» de l'espace de la rencontre thérapeutique
}

\section{René Raggenbass}

Médecin-Chef du Service de Médecine et de Psychiatrie dans les prisons valaisannes
Correspondance:

Dr René Raggenbass

Rue du Nord 4

CH-1920 Martigny

Tél. 0277224393

Fax 0277224493

rene.raggenbass@hin.ch
La médecine et la psychiatrie exercées dans le milieu carcéral suscite de vives polémiques qui impliquent les politiques, la justice, les services d'exécution des peines, les experts, les prévenus ou condamnés ainsi que le corps soignant [1].

Notre service de médecine et de psychiatrie, qui exerce dans les prisons valaisannes, est récent. Son entrée en activité date du $1^{\text {er }}$ janvier 2009. Il est composé d'une équipe d'une quinzaine de personnes dont des infirmiers/-ères, des psychologues cliniciennes, des médecins généralistes, un secrétariat et un psychiatre qui a la fonction de médecin-chef.

Je pourrais dire que cette petite équipe a pour mission, outre la prévention, de «guérir, soigner, apaiser la souffrance des corps et des esprits» [2], ceci dans un milieu où la logique de l'enfermement et de la sanction domine malgré la tâche de réinsertion qui incombe aussi à ces milieux. J'ajouterais encore que nos prestations doivent être équivalentes à celles prodiguées à la population générale.

La population carcérale du Valais comporte environ 300 prévenus/détenus/réfugiés [3]. Ce sont des femmes, des mineurs et des hommes de nationalités et de cultures différentes. Toutes les études menées dans ces milieux montrent que la morbidité tant

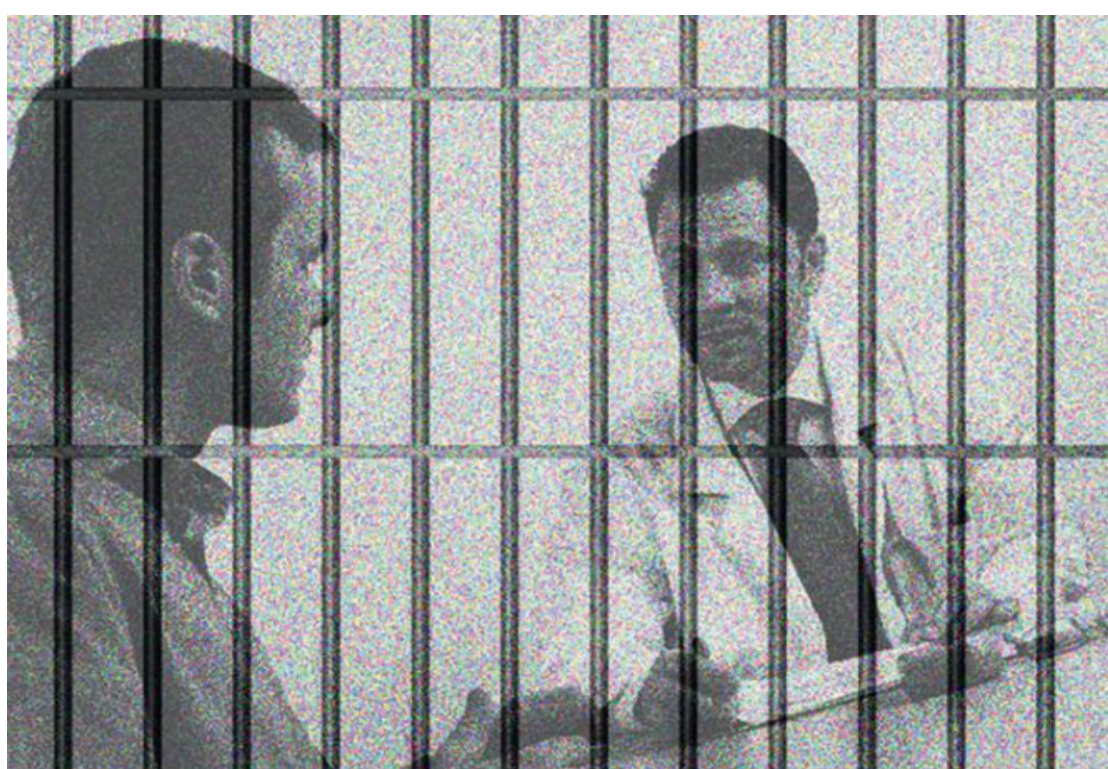

Depuis 2009 il existe un service de médecine et de psychiatrie, qui exerce dans les prisons valaisannes. somatique que psychiatrique y est, objectivement, plus élevée que dans la population générale.

C'est dans des circonstances très singulières où l'espace est limité, les déplacements contrôlés [4], la surveillance omniprésente [5], la peur des passages à l'acte obsédante et le secret médical (ou non) suspect, que notre Service doit faire sa place, une place différente, indépendante, à côté et articulée à, et non pas dans, la logique pénale. Pour l'autorité pénitentiaire et la justice, cette différenciation et cette indépendance ne vont pas de soi; pour ce qui nous concerne, notre liberté d'action n'est possible que parce que notre Service reste externe à l'administration pénitentiaire et qu'il se réfère directement à une administration sanitaire.

Cette position «à côté de», mais aussi «témoin de», génère évidemment beaucoup de tensions et d'incompréhensions dans le monde carcéral et judiciaire [6]. La justice, les autorités pénitentiaires ainsi que certaines spécialités de la médecine tentent de réduire la tension de cette différence en produisant des chimères modernes dont les signifiants sont: «la thérapie de justice» [7], «les experts forensiques», «les psychothérapeutes forensiques».

La chimère est une créature fantastique de l'Antiquité dont le corps est composé de plusieurs animaux. La chimère a été terrassée par Bellérophon, chevauchant son cheval ailé Pégase. Il y a plusieurs interprétations de ce mythe. La chimère représenterait les «désirs inassouvis, sources de frustration et plus tard de douleurs» (J. Chevalier, A. Gheerbrant) ou «la perversion des désirs matériels, la domination perverse sexuelle et le mensonge» (P. Diel) ou une figuration des cultes de la féminité et de ses prérogatives pour instaurer, par son meurtre, les nouveaux dieux masculins (R. Graves) [8]. Pour ce qui concerne les chimères modernes citées plus haut, je relèverais leur utopie et plus particulièrement le cynisme et l'impudence [9] de ceux qui les défendent.

Dans les soins pluriels du monde pénitentiaire, je souhaite me concentrer sur la logique du soin psychique que notre service met en place et soutient. Tout d'abord, notre travail ne consiste pas à faire taire nos patients ni à pratiquer une quelconque «orthopsyché». Il ne s'agit pas de rendre les détenus/ condamnés / réfugiés adaptés à l'enfermement. Comme le rappelle très justement Bertrand Kiefer, le travail de la médecine «n'est en aucun cas une entreprise au 
service d'une normalisation, ni d'une réintégration des individus déviants dans un chemin tracé» [10] j'ajouterais, sur le droit chemin.

Alors, quelle réponse notre service choisit-il d'offrir dans ce milieu où le sujet est, le plus souvent, si ce n'est toujours, réduit à la dimension, parfois horrible, de son acte? Cette fixation de la justice, du carcéral et du monde politique sur l'acte (que le clinicien nomme, entre autres, le passage à l'acte) a pour effet de forclore toute la dimension subjective de celui-ci. Cela justifie également les dérapages d'une psychia- thérapie est un processus, une dimension, qui peut survenir au cours de rencontres avec «un psy» mais qui peut tout aussi bien ne jamais advenir pour le sujet. L'instrument des acteurs est toujours une parole librement consentie. Ce n'est qu'à cette condition que le sujet pourra répondre de la «vérité interne» à son dire [14] une vérité qui n'a rien à voir avec celle que recherche la justice. Cela signifie que notre action partira toujours des dires du sujet, c'est-à-dire $\mathrm{du}$ cas par cas, et non pas d'un savoir, pour tous, à priori (ce qui est précisément le cas des experts) que

\section{«Mon objectif est de pointer et de soutenir la nécessité d'une position éthique du clinicien exerçant dans une prison.»}

trie qui cherche, comme au temps des augures, à se fonder sur la science de la prédiction. Le point commun de ces positions est de se référer au discours d'une pseudoscience [11] qui s'appuie sur les chimères citées plus haut, faute de pouvoir défendre la responsabilité éthique d'un discours qui leur soit propre [12].

Notre position se veut éthique. Cela signifie que nous ne reculons pas devant le Réel de l'acte criminel ni n'alimentons le «je n'en veux rien savoir» de la justice ou du sujet que nous rencontrons. Notre place est dans l'espace de la rencontre responsable avec le sujet. Cet espace permet une écoute privée, non surveillée, indépendante qui tient à laisser au sujet le temps et la liberté de déployer par la parole la logique de sa propre subjectivité quelque soit l'acte commis. Vous noterez que j'ai énoncé les termes d'espace et de rencontre et non pas de psychothérapie (ordonnée ou non) ce que confondent volontiers les interlocuteurs auxquels nous

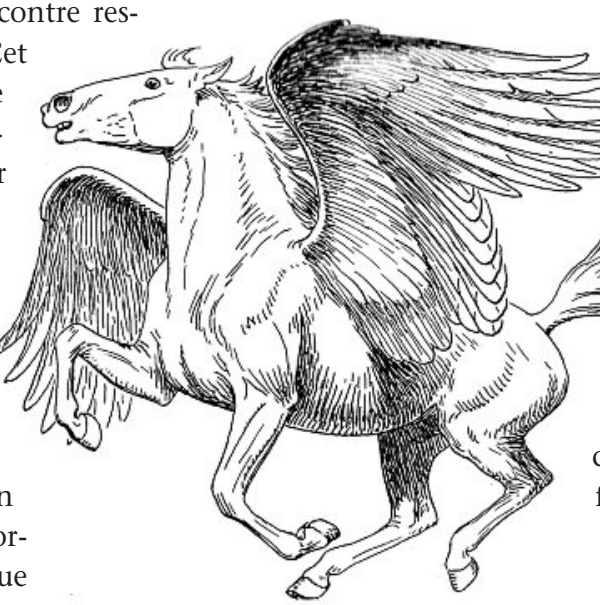

S'élever avec «Pégase» pour terrasser la chimère? nous supposerions avoir à son sujet. Il n'existe pas deux détenus qui parlent de la même manière de leur acte même si, pour la loi, l'acte est le même (vol, viol, meurtre, incendie...). Rappelons peut-être que nous nous révélons toujours dans les interstices de nos dires et de nos actes mais que de notre dire, nous sommes toujours responsables! C'est également le cas pour la personne détenue. Encore faut-il mettre à disposition l'espace, le temps et la liberté pour que ce dire, toujours singulier, puisse se déployer. C'est évidemment difficile lorsque l'urgence $\mathrm{du}$ «vouloir tout savoir sur l'acte» (pas sur le sujet!) et que la pression du «prévenir la récidive» occupe tout l'espace de pensée et de parole. Pourtant, c'est bien lorsque cette pression survient que nous avons le devoir éthique non seulement de proposer, mais aussi de défendre un lieu/espace privé, différencié de la logique pénale, qui fasse une place au sujet de l'acte reproché.

Il est évident que d'autres logiques comme celle de la criminologie ou d'autres mesures comme l'accompagnement éducatif, les encadrements socio-judicaires ou encore la surveillance socio-judiciaire ne doivent pas être abandonnées ni supprimées. Elles ont leur place dans la logique pénale mais il n'est en aucun cas possible de les fondre ni de les forcer dans le concept, le processus de psychothérapie ou encore dans l'acte thérapeutique quel que soit le modèle appliqué. Ces combinaisons de psychothérapies et de contrôle social sont des chimères qui donnent peut-être bonne conscience à ceux et à celles qui les créent mais qui ne satisfont en définitive personne: ni la justice, ni les politiques, ni 
les autorités pénitentiaires, ni les psychothérapeutes, ni encore les sujet concernés.

S'élever avec «Pégase» pour terrasser la chimère? Nous ne vivons pas dans une mythologie, ni ne sommes des dieux, mais nous savons tous que l'Humain peut révéler des dimensions cruellement réelles. C'est précisément parce que cette dimension existe chez nous tous que je crois fermement que notre responsabilité, notre position éthique de soignant dans les prisons réside non seulement à proposer, mais aussi à soutenir un objet-outil léger et ailé - celui d'un espace de rencontre thérapeutique indépendant et singulier - contre les chimères impudentes et pseudoscientifiquement fondées qu'il nous est de plus en plus souvent ordonné d'appliquer.

\section{Références}

1 Quelques exemples: L'ordre donné en Suisse en 2010 aux médecins par une autorité politique (Valais) et juridique (Tribunal Fédéral) de nourrir de force un détenu en grève de protestation, ayant toute sa capacité de discernement. Ou encore: La $6^{\text {bème }}$ conférence européenne pour la promotion de la santé en prison qui se tiendra à Genève du $1^{\text {er }}$ au 3 février 2011 et qui a pour thème: «Patients ou prisonniers? - Pistes vers l'équivalence des soins en prison». Ou encore: L'article du Prof. de droit, M. Müller, NZZ, 9 septembre 2010, qui déclare que «Le médecin des prisons est un fonctionnaire quelle que soit la modalité contractuelle qui l'engage dans cette activité. S'il n'obtempère pas aux ordres des autorités pénitentiaires, il doit en assumer les conséquences administratives» (trad. R. Raggenbass).

2 Badinter R. dans «Médecine, santé et Prison». Bertrand D et Niveau G. Ed. Genève: Médecine \& Hygiène; 2008. préface, p.XVI.

3 Pour les «réfugiés», il s'agit de personnes qui subissent une détention administrative dans l'attente d'une expulsion en raison de non-entrée en matière sur leur demande d'asile en Suisse (NEM).

4 Une dérive sécuritaire qui est qualifiée par plusieurs corps professionnels de «déshumanisante» (Libération du 10 mars 2010).

5 Wajcmann G. L'œil absolu. Ed Denoël ; 2010. Zeh J Trojanov I. Atteinte à la liberté; les dérives de l'obsession sécuritaires. Acte Sud. 2010.
6 Raggenbass R. L'inévitable ne se produit jamais, l'inattendu toujours. Conférence inédite donnée à la

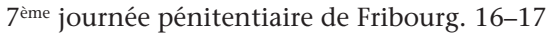
novembre 2010.

7 La thérapie de justice est une chimère qui tente d'unir la tête de la justice avec le corps des soignants. Son exercice ne vise pas en premier lieu le soin somatique ou psychique mais la réduction du risque de récidive de passage à l'acte délictueux. Brägger B. Gefängnismedizin in der Schweiz. Jusletter. 11 avril 2011. Cela dit, l'auteur de l'article, un juriste, n'explique pas pourquoi la LAMal, et non pas la justice elle-même, doit supporter les coûts de ces «thérapies de justice» qui n'ont pas pour objet le soin ni la guérison d'un trouble somatique ou psychique! Est-il encore légitime de les nommer «thérapies» alors qu'il s'agit en fait d'une mesure de contrôle social?

8 Wikipédia. «Chimère (mythologie)».

9 Le cynisme de cette impudence relève de «tous ceux dont les dires ne se sustentent ni d'un signifiant maître (le maître n'est pas impudent), ni d'un savoir assuré. Cela va des gourous de tout genre jusqu'aux experts de tout genre. [...] C'est qu'au maître on peut demander raison, alors que le savoir (le savoir du discours universitaire) est sans appel, il se dispense de toute justification et s'impose comme venant du réel, notamment quand il s'agit du vrai savoir de la science, celle qu'on dit dure.» Colette Soller, «Les affects lacaniens». PUF. 2011; pp. 94-5.

10 Le Bloc-notes de Bertrand Kiefer: «Prison et sauvagerie». Dans: Revue Médicale Suisse. Mars 2011. p. 728.

11 On lira avec intérêt: Psychanalyse, science et scientisme. Revue Mental. n²5 ; mars 2011.

12 C'est ainsi que certains politiques, juges et médecins démissionnent de leur champ éthique pour nous affliger d'un discours à l'idéologie pseudo-scientifique d'experts en tout genre (économiques, pénaux, sociaux et médicaux) dont les conséquences ne peuvent que nous inciter à nous préparer au pire. Les experts, nouvelles figures du sujet supposé savoir?

13 Ces deux thèmes méritent un développement spécifique.

14 Miller JA. La psychanalyse, sa place parmi les sciences. Dans: Mental. n²5; mars 2011. p. 17 